F. Costa, A. Monorchio, G. Manara, "Theory, design and perspectives of electromagnetic wave absorbers", IEEE Electromagnetic Compatibility Magazine, vol. 5, no. 2, pp. 67-74, August 2016.

doi: 10.1109/MEMC.0.7543954

URL: http://ieeexplore.ieee.org/abstract/document/7543954/ 


\title{
Theory, Design and Perspectives of Electromagnetic Wave Absorbers
}

\author{
Filippo Costa, Agostino Monorchio, Giuliano Manara \\ Dipartimento di Ingegneria dell'Informazione, University of Pisa \\ Email: filippo.costa(a.monorchio, g.manara)@iet.unipi.it
}

\begin{abstract}
Electromagnetic absorbers for free space and cavity absorption are discussed. Classical configurations are described as well as more recent designs. The presented layouts are able to provide absorbing behaviors spanning from ultra-narrow band to ultrawideband. A comparison among various solutions is presented both in terms of achievable bandwidth and in terms of minimum theoretical thickness. The problem of cavity resonances is also addressed. It is shown that resonances can be damped by using alternative solutions rather than the classic use of lossy magnetic materials.

Index Terms -Artificial Impedance Surface (AIS), Cavity resonances, Dallenbach layer, Electromagnetic Absorbers, Frequency Selective Surface (FSS), Jaumann screen, Metamaterials, Salisbury screen, Surface Wave Absorbers.
\end{abstract}

\section{INTRODUCTION}

Absorption reflection and transmission are the three fundamental properties of matter. The manner in which electromagnetic waves interact with an object depends upon the frequency of the radiation and the nature of the atoms of the object.

Electromagnetic absorbers are engineered structures, designed to increase absorption of electromagnetic waves across some desired portion of the spectrum with respect to that naturally achieved.

After some work in the thirties, the first strong development of this technology began with the World War II when Germany was interested into radar camouflage and United States wanted to improve performance of their radar by reducing interfering reflections from nearby objects [1]. German projects developed two kind of absorbing configurations: a semi flexible rubber sheet loaded with carbonyl iron powder and the Jauman absorber. On the other hand, the US project HARP (Halpern-anti-radar-paint) developed rubber sheets with resonant absorption behavior employing a high concentration of iron particles and the socalled Salisbury screen absorber [2]. Interest in a practical version of such an arrangement spread rapidly due to its simplicity and scalability across frequency spectrum. The development of absorbing walls was also the input for the first anechoic chambers [1].

Salisbury screen structure consists of a resistive sheet having a. resistance of $377 \mathrm{ohm} / \mathrm{sq}$ located a quarter-wavelength out from a reflecting surface. The Jaumann absorber can be conceptually considered an extension of the Salisbury screen since it consists of two or more resistive sheets separated by a distance of approximately $\lambda / 4$, thus providing a broadband response.

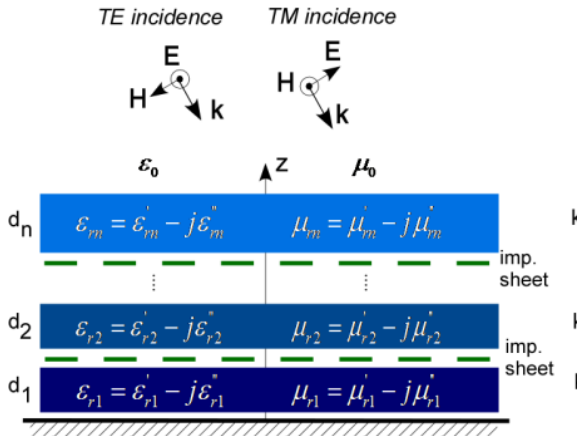

(a)

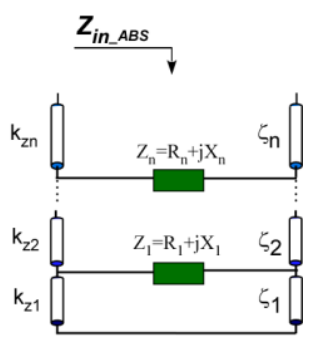

(b)
Fig. 1 - .(a) Layout of a general absorbing structure and its transmission line equivalent circuit (b). Salisbury screen, Jaumann screen, Dallenbach layer and Circuit analogue absorbers (CCA) and different FSS-based absorbers are particular cases of the represented stratified structure.

Absorbers comprising a single high-lossy layer in front of a ground plane are known as Dällenbach layer [3]. Several materials with different chemical properties can be employed for this design but the final goal is to obtain that the imaginary portions of the electric permittivity and the magnetic permeability are adequately tuned to achieve the desired absorption. Frequency Selective Surfaces (FSSs), that are planar periodic arrays formed by the repetition of scatters with an arbitrary geometry, can also profitably be employed to design both narrowband and wideband electromagnetic absorbers. Narrowband absorbers can be synthesized having thickness much thinner than a quarter wavelength by exploiting the capacitive behavior of the FSS impedance. Ultra-narrow band configurations are also known as perfect metamaterial absorbers because of their very angularly stable absorption behavior at a single wavelength. Single layer employing resistive FSSs can be designed to achieve wideband absorption with a reduced thickness. This configuration, also known as Circuit Analog (CA) absorber, can be considered an extension of the Salisbury screen [4], [5]. Stacking an increasing number of capacitive FSS layers [6]-[8] is also a powerful method to improving absorbing performance of classical Jaumnan configuration.

Applications of absorbing materials span from their use as a fundamental building block in designing novel devices such as power imaging devices [9], photodetectors, microbolometers [10], [11] phase modulators [12] or frequency selective emitters [13], [14] and their employment 


\section{IEEE Electromagnetic Compatibility (EMC) Magazine, PREPRINT}

in a wide range of scenarios for improving the performance of existing technologies. Some key applications across microwave frequencies are the reduction of radar signature of targets [15]-[19] and the reduction of electromagnetic interference (EMI) in complex scenarios. While the former application is mainly related to radar counter measurement, the latter is of particular interest for the electromagnetic compatibility community. Improving electromagnetic compatibility implies, for example, reducing radiation from nearby reflecting objects that can cause spurious signals (multipath reduction), minimize mutual coupling between closely placed antennas, reducing harmful effects due to cavity resonance in enclosed monolithic microwave integrated circuits (MMIC) circuits [20], [21], mitigating the effect of apertures in metallic cavities [22] and attenuating surface waves [23]-[26]. Avoiding multiple reflections is, for instance, strategic for achieving a correct reading in Radio Frequency Identification (RFID) [27].

Electromagnetic absorbers design is usually driven by the concept of impedance match to free space. When the absorber fulfills this condition, the impinging electromagnetic waves do not find any impedance discontinuity when they encounter the absorbing layer and thus electromagnetic energy is converted into heat. However, when absorbers are employed for damping cavity resonances or absorbing surface waves, the matching approach is not appropriate. In those scenarios, it is necessary to analyze and solve the dispersion equation trying to maximize, via the use of the most suitable absorbing layer, the attenuation factor of the supported mode [20], [25].

The paper is organized as follows. In the next section the matching approach to absorb electromagnetic waves is introduced. In section III the absorbing configurations are briefly introduced. In section IV, some guidelines to obtain wideband behaviors are presented. Section V is dedicated to the comparison of the absorbing performance among the presented configurations. Finally, the problem of damping cavity resonances is addressed in section VI.

\section{PLANE WAVE ABSORPTION: MATCHING APPROACH}

Electromagnetic absorbers can be efficiently analyzed by using the equivalent transmission-line (TL) method [28]. When a plane wave impinges upon the surface of the absorber, normally or obliquely, the amount of reflected power is determined by the mismatch between free space and absorber impedance. For absorbing surfaces backed by an impenetrable surface there is not transmitted energy and, as a consequence, the power that is not reflected, is dissipated in the lossy structure. The magnitude of the reflection coefficient of generic absorbing surface reads:

$$
\left|\Gamma^{T E, T M}\right|=\sqrt{\frac{\left(\operatorname{Re}\left\{Z_{\text {in }}^{T E, T M}\right\}-\zeta_{0}^{T E, T M}\right)^{2}+\left(\operatorname{Im}\left\{Z_{\text {in }}^{T E, T M S}\right\}\right)^{2}}{\left(\operatorname{Re}\left\{Z_{\text {in }}^{T E, T M}\right\}+\zeta_{0}^{T E, T M}\right)^{2}+\left(\operatorname{Im}\left\{Z_{\text {in }}^{T E, T M S}\right\}\right)^{2}}}
$$

where $Z_{\text {in_ABS }}$ and $\zeta_{0}$ represent the input impedance of the absorbing structure and the characteristic impedance of free space for TE or TM polarization, respectively. For a lossless structure, the real part of the input impedance is null and the reflection coefficient magnitude always equals the unity. As lossy substrates or lossy surfaces are employed in the design, the real part of the input impedance $Z_{\text {in_ABS }}$ decreases. The losses can be introduced in the dielectric substrate (dielectric losses) or in a surface (ohmic losses). The former is the case of the Dallenbach layer absorbers [29]-[31] and the latter is the case of Salisbury, Jaumann or CCA absorbers [8], [32][34]. In order to achieve a good absorption level at a single frequency or over a wide frequency band, it is necessary to create one or more resonances where the imaginary part of the input impedance is zero. At the same time, the real part should be matched to the free space impedance which is equal to $\zeta_{0} / \cos (\theta)$ and $\zeta_{0} \cos (\theta)$ for TE and TM polarization, respectively ( $\theta$ represents the incident angle).

The design of the absorber can be accomplished by obtaining a closed form expression of both the real and imaginary parts of the input impedance of the absorber [34]-[37] and then imposing the following conditions:

$$
\begin{aligned}
& \operatorname{Re}\left\{Z_{i n_{-} A B S}\right\}=\varsigma_{0}^{T E, T M} ; \\
& \operatorname{Im}\left\{Z_{\text {in_ABS }}\right\}=0
\end{aligned}
$$

Another approach consists in solving the analytic transmission line relations representing the input impedance of the absorber through a parametric graphical solutions [30], [38]. The least-elegant one, but sometimes sufficient, is the use of an iterative or evolutionary optimization algorithm without resorting to the analysis of physical phenomena under investigation [39], [40].

The input impedance of a generic single layer or multilayer absorbing structure, is computed with classical transmission line approach eventually connecting lumped purely real (in case of resistive sheets) or complex impedances (in case of periodic surfaces) in parallel according to the model depicted in Fig. 1.

\section{ABSORBING CONFIGURATIONS}

In this section, we briefly introduce the classical absorbing configurations and more recent designs based on Frequency Selective Surfaces (FSS).

\section{a) Dallenbach layer}

The use of a single lossy substrate backed by a ground plane is the simpler strategy to achieve absorption of electromagnetic waves. Most of the commercially available absorbing materials are designed by using this approach [41], [42] because of its mechanical robustness. Usually magnetically loaded, silicone rubber materials or low-loss dielectric foam or resins are employed by industry. The use of magnetic materials brings reduced thickness and wide absorption bands but the improvements are counterbalanced in terms of weight and cost. A magnetically loaded panel of dimensions $30 \mathrm{~cm} \times 30 \mathrm{~cm}$ weighs around $1 \mathrm{~kg}$. From a TL point of view, the input impedance of the single absorbing layer is represented by a short-circuited transmission line:

$$
Z_{i n_{-} A B S}^{T E, T M}=j Z_{1}^{T E, T M} \tan \left(k_{z 1} d_{1}\right)
$$




\section{IEEE Electromagnetic Compatibility (EMC) Magazine, PREPRINT}

If the absorber is made by more than one layer, the transmission lines are cascaded by using standard transmission line theory [43]. The minimization of (3), at a single frequency or over a wide band, can be addressed via a parametric analysis or an optimization algorithm. However, it is important to highlight that it is not possible to manufacture materials with an arbitrary value of dielectric permittivity and magnetic permeability over a wide frequency range because of physically dispersive behavior of natural materials. For this reason, the design optimization process is usually constrained within a set of realistic materials, preliminarily characterized [44], [45].

\section{b) Salisbury screen}

The Salisbury screen is a well-known absorbing configuration composed by a resistive sheet on top of a metallic plane [35]. It is based on a simple assumption, that is, the input impedance of the absorber is determined by the surface resistance of the resistive sheet, if the resistive sheet is accommodated a quarter wavelength apart from a ground plane. Indeed, the input impedance of the absorber is given by the parallel connection between the input impedance of the grounded substrate (which tends to infinite at a distance of a quarter wavelength from a ground plane) and the resistive impedance of the sheet. Being a resonant configuration, the operating bandwidth is limited around the resonance frequency.

\section{c) Jaumann screen}

Jaumann screen can be viewed as an extension of the Salisbury absorber having resistive sheets placed at a distance of a quarter wavelength from each other [29]. An important characteristic of the layers is that their sheet resistance must be gradually increased while moving away from the ground plane. In this way, the electromagnetic wave gradually penetrates inside the absorbing structure encountering a low reflection in the outer layers of the structure. For this reason the Jaumann absorber can be also seen as a graded absorber [46]. This configuration can be employed to design wideband absorbers. The larger are the number of layers, the wider is the obtained bandwidth. Apparently, bandwidth enlargement has a counterpart in terms of thickness and thus of mechanical robustness.

\section{d) Absorbers based on FSSs}

Periodic surfaces or frequency selective surfaces are also largely employed in the literature to design electromagnetic absorbing surfaces [4], [8], [33], [34], [47]-[50]. The added value of FSS with respect to simple resistive sheets is that the impedance of a periodic surface is not purely real but complex. This reactive part can be efficiently exploited to generate additional resonant behaviors in the stratified structure. A simple layout allowing the synthesis of both ultra-narrowband, narrowband, wideband electrically-thin absorbers is the Artificial Impedance Surface (AIS) which comprises an FSS with suitably shaped periodic elements on top of a thin grounded low-loss dielectric slab.

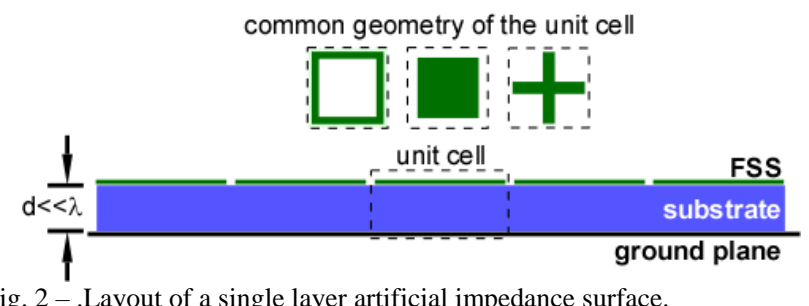

Fig. 2 - .Layout of a single layer artificial impedance surface.

The layout of this absorber, which is shown in Fig. 2, can be seen as an evolution of the classical Salisbury screen and, similarly to it, it does not employ magnetic materials being characterized by a very low weight. The AIS can be seen as a parallel LC circuit [51] at the fundamental resonance: it resonates when the capacitive impedance value of the FSS assumes the same value of the inductive impedance of the grounded substrate but with opposite sign.

Absorption from the AIS surface is achieved if the substrate and/or the FSS are characterized by a suitable amount of losses and a proper thickness. Indeed, the real part of the input impedance needs to be matched to the free space impedance at the resonance. The input impedance of a lossy AIS, at the fundamental resonance, can be computed in an approximate closed form [36]. The imaginary part of the input impedance $Z_{\text {in_ABS }}$ crosses the zero and the real part reads [36], [37]:

$$
\operatorname{Re}\left\{Z_{\text {in_ABS }}\right\} \approx \frac{\left(\operatorname{Im}\left\{Z_{d}\right\}\right)^{2}}{\left(\operatorname{Re}\left\{Z_{d}\right\}+R_{O}+R_{D}\right)}
$$

where $R_{O}$ and $R_{D}$ takes into account dielectric and ohmic losses due to the lossy FSS and $Z_{d}$ is the input impedance of the low-loss grounded substrate as defined in (3). The expression in (4) contains all the degrees of freedom of the single resonant AIS absorber: it is a function of the FSS reactance (inside $R_{D}$ ), of the electrical substrate thickness (inside $Z_{d}$ ) and of the real and imaginary part of the dielectric permittivity (inside $R_{D}$ and $Z_{d}$ ).

Depending on the prevalent nature of losses (they can be ohmic if the FSS is lossy or dielectric if the substrate is highly lossy and the FSS is metallic) a classification of absorbing configurations can be defined [52]:

- $R_{D}>>R_{O}$ : Ohmic losses negligible with respect to dielectric losses. Metallic FSS printed on an extremely thin low-loss dielectric slab. This structure is frequently referred to as perfect metamaterial absorber because of its peculiarity of absorbing impinging electromagnetic waves from all angles in a very small bandwidth.

- $R_{D} \approx R_{O}$ : Both ohmic and dielectric losses contribute to absorption at the resonance. The structure is typically narrowband.

- $R_{O}>>R_{D}$ : Ohmic losses are predominant. It is typically the case of FSSs made with resistive materials. The structure is typically wideband or ultra-wideband if a multilayer capacitive FSS configuration is adopted [8].

Lossy surfaces can be synthesized either by employing resistors in each unit cell of the periodic surface on a metallic 


\section{IEEE Electromagnetic Compatibility (EMC) Magazine, PREPRINT}

surface [53]-[55] or by printing periodic shapes using resistive inks [34].

\section{SYNTHESIS OF ABSORBERS EMPLOYING FSSS}

As previously remarked, synthesis of narrowband absorbers by employing the AIS surfaces can be achieved when the inductive input impedance of the grounded substrate and the capacitive FSS impedance have the same value but opposite signs and, at the same time, a suitable amount of losses are included in the structure. If the AIS surface is suitably designed, an additional resonance can be introduced in order to drastically enlarge the operating bandwidth. The second resonance is obtained if the capacitive impedance of the grounded substrate (thicker than a quarter wavelength) will be equal to the inductive impedance of the FSS (after its proper resonance). At the center of the operating band the input impedance of the substrate tends to infinite and the FSS impedance can be designed to have a purely real impedance with zero imaginary part. The optimal surface resistance can be derived from the equivalent circuit model [34], [56]. The working principle of the structure can be easily understood by tracing the impedance curves on a Cartesian plot as shown in Fig. 3a.
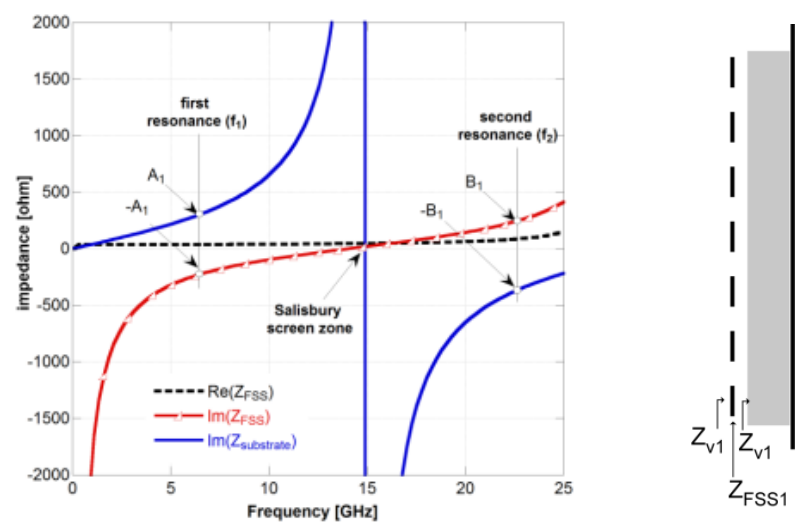

(a)
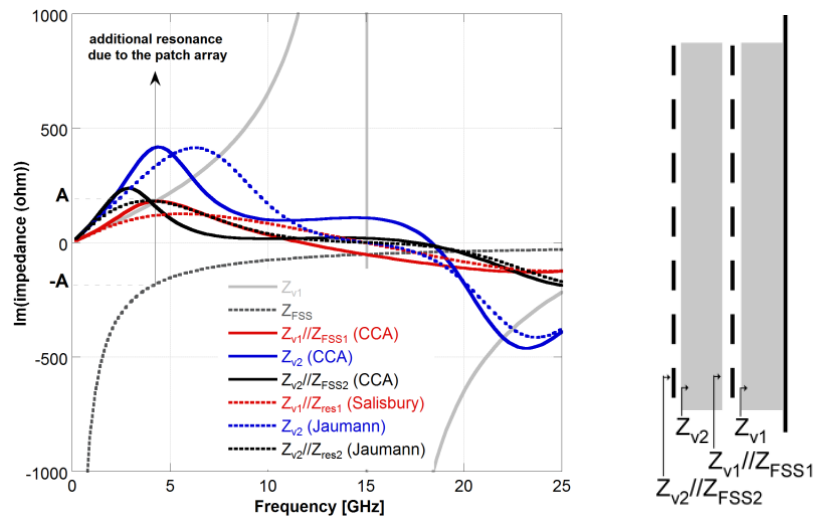

(b)

Fig. 3 - .(a) Impedance behavior of a single layer FSS absorber. (b). Input impedance at various level of the stack up both for Jaumann screen and for capacitive circuit absorber (CCA). The capacitive impedance of the FSS allows to strongly enlarge the absorption bandwidth at low-frequencies.
If multiple FSS layers are stacked on top of each other with a configuration similar to the Jaumann absorber (uniform resistive sheets are replaced by the FSSs layers), ultrawideband absorbers can be also obtained. In the design of ultra-wideband absorber, the unit cell periodicity should be small enough to avoid the propagation of grating lobes (additional propagation directions [33]) at the highest absorption frequency. For this reason, sub-wavelength lowpass arrays are employed instead of the conventional resonating FSS elements [7], [8]. The low-pass arrays have capacitive impedance over the whole frequency band and, when they are made of resistive materials, they can be modeled by series RC circuits over the entire frequency band of interest. Capacitive circuit method is also powerful in the optimization of oblique incidence since patch arrays can be modeled accurately for different incident angles and polarizations [57]. Another important parameter in designing absorbers for oblique angle of incidence is to employ proper dielectric layers since they can provide scan compensation, bandwidth increase and frequency response stabilizations [6]. In order to understand the effect obtained by replacing the resistive sheets typical of Jaumann configuration with arrays of resistive patches, the impedance of both the Jaumann absorber and the capacitive circuit absorber (CCA) are analyzed. A simple dual layer structure is considered for simplicity. The impedances at various interfaces are reported in Fig. 3b. It is apparent that the imaginary part of the FSS impedance allows introducing an additional parallel resonance thus improving the smoothness of the imaginary part of the absorber input impedance. This allows improving the low frequency behavior of the multilayer.

\section{COMPARISON BETWEEN ABSORBING CONFIGURATIONS}

Narrowband configurations are initially discussed. The typical reflection performance of previously mentioned configurations are reported in Fig. 4.

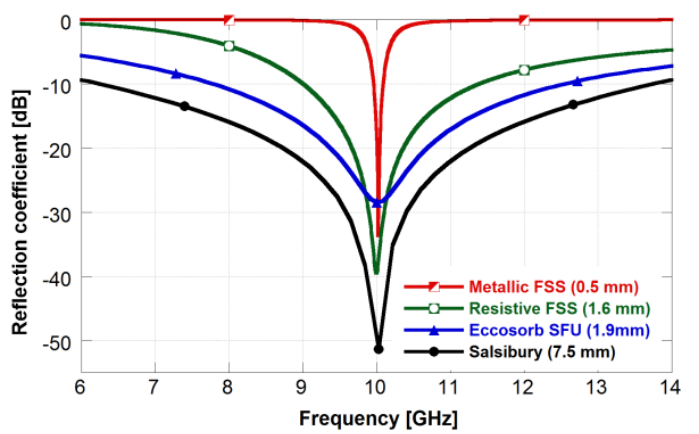

Fig. 4 - .Reflection coefficient of four different narrowband absorbing configurations. Absorbers employing FSSs and magnetic loaded (Eccosorb) are thinner than a quarter wavelength.

Metamaterial absorbers employing metallic FSS can be made arbitrarily thin by presenting very good absorption performance at the resonant frequency. This absorption profile is also very stable with the incident angle. The absorption bandwidth is strongly dependent upon substrate thickness and magnetic permeability [58]. For this reason, magnetic loaded absorber (for instance Eccosorb SFU10) 


\section{IEEE Electromagnetic Compatibility (EMC) Magazine, PREPRINT}

guarantees a large bandwidth with a small thickness. Salisbury is the most wideband among the reported configurations, but it is one of the thickest one since it resonates with a quarter-wavelength substrate.

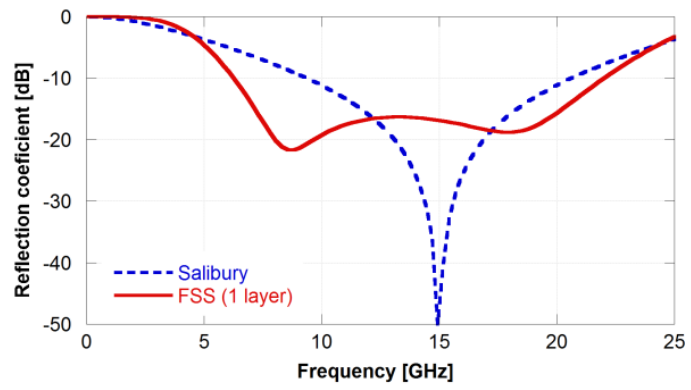

(a)

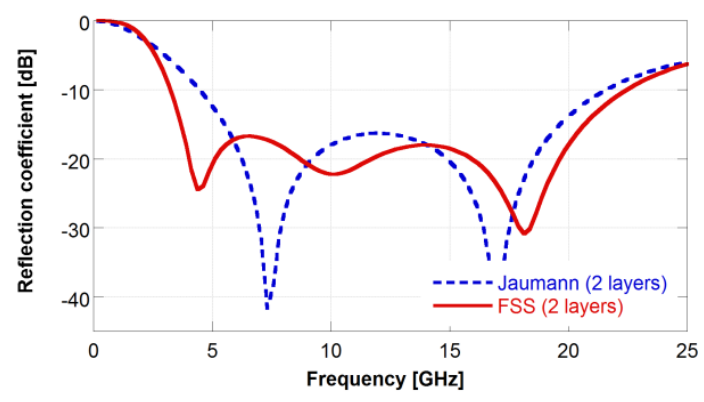

(b)

Fig. 5 - .(a) Reflection coefficient of a $5 \mathrm{~mm}$ Salisbury screen compared with a $5 \mathrm{~mm}$ AIS absorber employing an array of resistive loops. (b). Reflection coefficient of a $12 \mathrm{~mm}$ Jaumann screen compared with a $12 \mathrm{~mm}$ Capacitive Circuit Absorber (CCA) employing an array of resistive patches. Parameters of Jaumann and CCA are the same: $\mathrm{d}_{1}=\mathrm{d}_{2}=\mathrm{d}_{3}=4 \mathrm{~mm} \varepsilon_{\mathrm{r} 1}=1 ; \varepsilon_{\mathrm{r} 2}=$ $\varepsilon_{\mathrm{r} 3}=2 . \mathrm{R}_{\mathrm{s} 1}=150 \Omega / \mathrm{sq} ., \mathrm{R}_{\mathrm{s} 2}=500 \Omega / \mathrm{sq}$.

In order to compare the performance of Salisbury and Jaumann configurations with those employing periodic surfaces, the following structures are compared:

- a $\lambda / 4$ Salisbury screen with $5 \mathrm{~mm}$ air substrate is compared with an AIS absorber with the same thickness employing an array of resistive loops having a surface impedance of $70 \Omega / \mathrm{sq}$;

- a Jaumann screen composed by 3 dielectric substrates of $4 \mathrm{~mm}$ (total thickness of $12 \mathrm{~mm}$ ) with two resistive sheets in between is compared with a capacitive circuit absorber having the same dielectric stack up. The only difference is that the uniform resistive sheets are replaced by two arrays of patches characterized by the same surface resistance.

The reflection coefficient achieved with the aforementioned configurations are reported in Fig. 5. It is apparent that the presence of the FSS layer helps in increasing the absorption without impacting the total thickness and leaving the stack up unaltered.

The main objective in the design of an absorber is to get the largest possible bandwidth with a small thickness. Rozanov has shown that, for any metal-backed absorber, the infinite integral of the reflectance is bounded under certain conditions [58]. According to [58] the minimum thickness for achieving a certain absorption profile $R$, is given by:

$$
d \geq \frac{\left|\int_{0}^{\infty} \ln \right| R(\lambda)|d \lambda|}{\mu_{r} 2 \pi^{2}}
$$

where $\lambda$ is the wavelength in free space and $\mu_{r}$ is the static permeability of the layer. The minimum possible thickness can be used as a parameter to judge the efficiency of a design method. Some of the above analyzed configurations are compared against the minimum theoretical thickness calculated according to (5). The integral in (5) is computed by considering the actual profiles shown in Fig. 5 . The results are summarized in Table 1.

Table 1 - Electrical and geometrical parameters of the analyzed wideband and ultra-wideband absorbing structures. The physical limit is computed according to (5).

\begin{tabular}{lcccc}
\hline Configuration & $\begin{array}{c}\text { A: } \\
\text { Thick- } \\
\text { ness } \\
{[\mathbf{m m}]}\end{array}$ & $\begin{array}{c}\text { B: } \\
\text { Rozanov } \\
\text { Limit } \\
{[\mathbf{m m}]}\end{array}$ & $\begin{array}{c}\text { Physical } \\
\text { limit/ } \\
\text { thickness } \\
\left(\mathbf{A} / \mathbf{B}^{* 100}\right)\end{array}$ & $\begin{array}{c}\text { Bandwidth \% } \\
(\mathbf{- 1 5} \mathbf{~ d B}) \\
\mathbf{2}\left(\mathbf{f}_{\max }-\mathbf{f}_{\text {min }}\right) / \\
\left(\mathbf{f}_{\max }+\mathbf{f}_{\text {min }}\right)\end{array}$ \\
\hline Salisbury & 5 & 4.4 & 88 & 45.4 \\
FSS (1 layer) & 5 & 4.48 & 89.6 & 97.1 \\
$\begin{array}{l}\text { Jaumann } \\
\text { (2 layers) }\end{array}$ & 12 & 10.68 & 89 & 111.9 \\
$\begin{array}{l}\text { Capacitive } \\
\text { FSS (2 layers) }\end{array}$ & 12 & 11.05 & 92.1 & 140.2 \\
\hline
\end{tabular}

\section{ABSORPTION OF CAVITY MODES: DISPERSION EQUATION APPROACH}

Many circuits, especially monolithic microwave integrated circuits (MMIC), are accommodated in metal packages to provide mechanical support, power routing, signal distribution, thermal dissipation and shielding. However, these cavities support resonant modes at the frequencies where circuits operates. Resonant modes have usually a very high quality factor $\mathrm{Q}$ and even a very loose coupling between the circuit and these modes can disturb circuit operation. Microwave-absorbing materials are a demonstrated and viable method for damping cavity modes. The energy is usually attenuated with lossy magnetic introduced into the cavity [21].

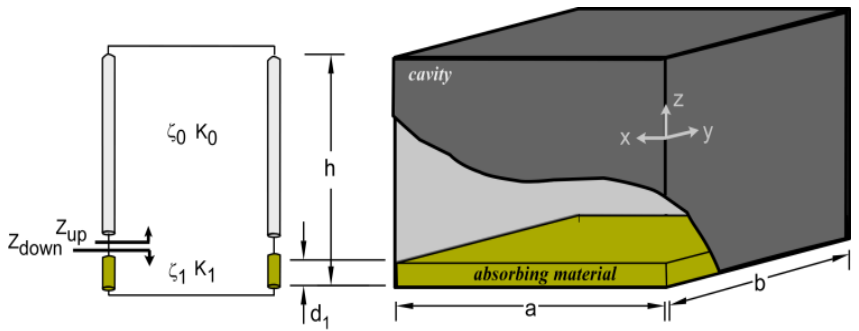

Fig. 6 - Three dimensional sketch of a resonance cavity loaded with a magnetic lossy substrate and it equivalent transmission line.

However, it has to be noted that microwave absorbers based on organic materials, such as silicon rubber, outgas significant amount of hydrogen which could compromise the reliability of the MMIC enclosed within the package [59]. 


\section{IEEE Electromagnetic Compatibility (EMC) Magazine, PREPRINT}

Absorbing materials with resistive sheets do not pose reliability problem for the circuits.

The previously introduced approach based on impedance matching is not appropriate to face the problem of cavity resonance attenuation. Indeed, in this case, absorbers should be designed to attenuate guided modes not plane waves.

The problem can still be faced by using the transmission line equivalent and deriving the dispersion equation of the cavity loaded with a lossy dielectric slab. Let us consider the rectangular cavity reported in Fig. 6. We impose the continuity of the voltages and currents at a generic interface [60], thus the sum of $\mathrm{Z}_{\mathrm{up}}$ and $\mathrm{Z}_{\text {down }}$ equal to zero:

$$
Z_{1}^{T E, T M} \tan \left(k_{z 1} d_{1}\right)+Z_{0}^{T E, T M} \tan \left(k_{z}\left(h-d_{1}\right)\right)=0
$$

where $Z_{l}^{T E}=\left(\omega \mu_{r} \mu_{0}\right) / \beta ; \quad Z_{l}^{T M}=\beta /\left(\omega \varepsilon_{r} \varepsilon_{0}\right)$ are the characteristic impedances of the slab for TE and TM polarization, $k_{z l}=\sqrt{k_{0}{ }^{2} \varepsilon_{r} \mu_{r}-k_{t(m, n)}^{2}}$ is the propagation constant along the normal unit of the slab of the $l^{\text {th }}$ layer and $k_{t(m, n)}=\sqrt{\left(\frac{n \pi}{a}\right)^{2}+\left(\frac{m \pi}{b}\right)^{2}}$ is the transverse wavenumber within the cavity. Solving the dispersion equation (6) allows to calculate the resonance frequencies of the loaded cavity. In presence of lossy materials, the resonance frequencies are complex $\left(\omega_{\mathrm{r}}+\mathrm{j} \omega_{\mathrm{i}}\right)$ and the imaginary part of them represents the damping factor of the specific mode. As a consequence the quality factor of the mode can be estimated as [20]:

$$
Q \approx \frac{\omega_{r}}{2 \omega_{i}}
$$

Considering that both $\varepsilon_{r}$ and $\mu_{r}$ are complex numbers, it is not possible to solve the transcendental equation in a closed form but it is possible to find the resonance frequencies numerically. In the presence of high lossy materials, the solutions are in the complex plane far from the real axis. For this reason the roots are searched iteratively by increasing losses gradually [61].

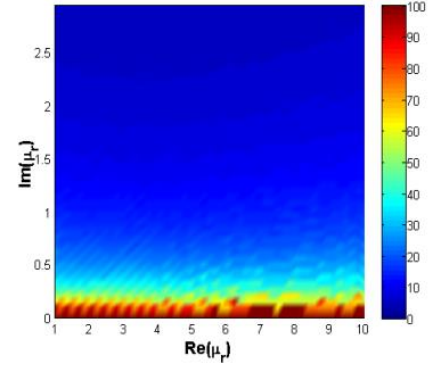

(a)

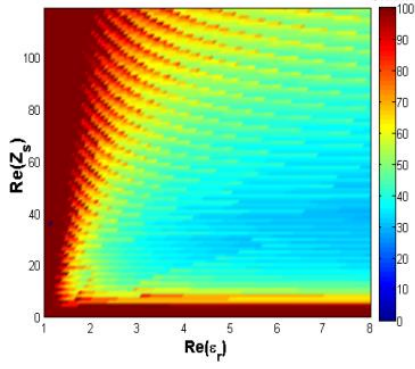

(b)
Fig. 7 - Quality factor of the $\mathrm{TM}_{110}$ mode of the cavity loaded with lossy magnetic material (a) low-loss dielectric slab covered by a resistive sheet

As a practical example, a realistic cavity $(a=200 \mathrm{~mm}, \mathrm{~b}=100$ $\mathrm{mm}, \mathrm{h}=50 \mathrm{~mm}$ ) is considered. The fundamental mode for this cavity is the $\mathrm{TM}_{110}$ (we are considering propagation along $\mathrm{z}$ direction). The unloaded $\mathrm{Q}$ factor of the mode is above 2000. The cavity is then loaded with a lossy substrate of $3 \mathrm{~mm}$. The new Q-factor is computed according to (7) by varying both the real and the imaginary part of magnetic permeability. The same analysis is carried out by loading the cavity with a $3 \mathrm{~mm}$ thick low-loss dielectric substrate covered by a resistive sheet. The new $\mathrm{Q}$ factors are found as a function of the relative dielectric permittivity of the substrate and of the surface resistance of the resistive sheet. The results of both of the analyses are reported in Fig. 7. It is apparent that the loading with magnetic lossy materials is an effective mean to damping cavity resonance. However, also the use of a resistive sheet allows to drastically reduce the field within the cavity without using magnetic substrates.

\section{CONCLUSIONS}

Classical absorbing configurations and FSS-based absorbers have been discussed and compared. The latter configuration can provide ultra-narrow band absorbers (metamaterial absorber), narrowband, wideband (artificial impedance surface absorbers) and ultra-wideband behavior (capacitive circuit absorber) with better performance with respect to the classical Salisbury and Jaumann configurations. The problem of cavity resonance reduction is also addressed showing that resistive sheets can be employed to damping cavity resonances as a valid alternative to magnetic materials.

\section{REFERENCES}

[1] W. Emerson, "Electromagnetic wave absorbers and anechoic chambers through the years," IEEE Trans. Antennas Propag., vol. 21, no. 4, pp. 484-490, Jul. 1973

[2] W. W. Salisbury, "Absorbent body for electromagnetic waves," US2599944 A.

[3] W. Dallenbach and W. Kleinsteuber, "Reflection and Absorption of Decimeter-Waves by Plane Dielectric Layers," Hochfreq. u Elektroak, pp. 152-156, 1938.

[4] D. Sjöberg, "Analysis of wave propagation in stratified structures using circuit analogues, with application to electromagnetic absorbers," Eur. J. Phys., vol. 29, no. 4, p. 721, 2008.

[5] L. Sun, H. Cheng, Y. Zhou, and J. Wang, "Broadband metamaterial absorber based on coupling resistive frequency selective surface," Opt. Express, vol. 20, no. 4, pp. 4675-4680, 2012.

[6] A. Kazemzadeh and A. Karlsson, "Multilayered wideband absorbers for oblique angle of incidence," IEEE Trans. Antennas Propag., vol. 58, no. 11, pp. 3637-3646, 2010.

[7] A. Kazemzadeh, "Nonmagnetic ultrawideband absorber with optimal thickness," IEEE Trans. Antennas Propag., vol. 59, no. 1, pp. 135-140, 2011.

[8] A. K. Zadeh and A. Karlsson, "Capacitive circuit method for fast and efficient design of wideband radar absorbers," IEEE Trans. Antennas Propag., vol. 57, no. 8, pp. 2307-2314, 2009.

[9] S. Yagitani, K. Katsuda, M. Nojima, Y. Yoshimura, and H. Sugiura, "Imaging radio-frequency power distributions by an EBG absorber," IEICE Trans. Commun., vol. 94, no. 8, pp. 2306-2315, 2011.

[10] T. Maier and H. Brückl, "Wavelength-tunable microbolometers with metamaterial absorbers," Opt. Lett., vol. 34, no. 19, pp. 3012-3014, 2009.

[11] S. A. Kuznetsov, A. G. Paulish, A. V. Gelfand, P. A. Lazorskiy, and V. N. Fedorinin, "Bolometric THz-to-IR converter for terahertz imaging," Appl. Phys. Lett., vol. 99, no. 2, p. 023501, 2011.

[12] H.-T. Chen, W. J. Padilla, M. J. Cich, A. K. Azad, R. D. Averitt, and A. J. Taylor, "A metamaterial solid-state terahertz phase modulator," Nat. Photonics, vol. 3, no. 3, pp. 148-151, 2009.

[13] X. Liu, T. Tyler, T. Starr, A. F. Starr, N. M. Jokerst, and W. J. Padilla, "Taming the blackbody with infrared metamaterials as selective thermal emitters," Phys. Rev. Lett., vol. 107, no. 4, p. 045901, 2011.

[14] J.-J. Greffet, "Applied physics: Controlled incandescence," Nature, vol. 478, no. 7368, pp. 191-192, 2011. 


\section{IEEE Electromagnetic Compatibility (EMC) Magazine, PREPRINT}

[15] H.-K. Jang, J.-H. Shin, and C.-G. Kim, "Low RCS patch array antenna with electromagnetic bandgap using a conducting polymer," in International Conference on Electromagnetics in Advanced Applications (ICEAA), 2010, 2010, pp. 140-143.

[16] F. Costa and A. Monorchio, "A frequency selective radome with wideband absorbing properties," IEEE Trans. Antennas Propag., vol. 60 , no. 6, pp. 2740-2747, 2012.

[17] F. Costa, S. Genovesi, and A. Monorchio, "A frequency selective absorbing ground plane for low-RCS microstrip antenna arrays," Prog. Electromagn. Res., vol. 126, pp. 317-332, 2012.

[18] S. Genovesi, F. Costa, and A. Monorchio, "Low-profile array with reduced radar cross section by using hybrid frequency selective surfaces," IEEE Trans. Antennas Propag., vol. 60, no. 5, pp. $2327-$ 2335, 2012.

[19] S. Genovesi, F. Costa, and A. Monorchio, "Wideband Radar Cross Section Reduction of Slot Antennas Arrays," IEEE Trans. Antennas Propag., vol. 62, no. 1, pp. 163-173, Jan. 2014.

[20] D. F. Williams, "Damping of the resonant modes of a rectangular metal package [MMICs]," IEEE Trans. Microw. Theory Tech., vol. 37, no. 1, pp. 253-256, Jan. 1989.

[21] P. Dixon, "Cavity-resonance dampening," IEEE Microw. Mag., vol. 6, no. 2, pp. 74-84, Jun. 2005.

[22] R. Araneo, G. Lovat, and S. Celozzi, "Low-frequency intertwined spiral-aperture absorbers for Shielded enclosures," in 2013 IEEE International Symposium on Electromagnetic Compatibility (EMC), 2013, pp. 456-461.

[23] R. T. Ling, J. D. Scholler, and P. Y. Ufimtsev, "The Propagation and Excitation of Surface Waves in an Absorbing Layer - Abstract," $J$. Electromagn. Waves Appl., vol. 12, no. 7, pp. 883-884, Jan. 1998

[24] D. Shively, "Surface waves on a grounded dielectric slab covered by a resistive sheet," IEEE Trans. Antennas Propag., vol. 41, no. 3, pp. 348-350, Mar. 1993.

[25] P. Y. Ufimtsev and R. T. Ling, "New results for the properties of TE surface waves in absorbing layers," IEEE Trans. Antennas Propag., vol. 49, no. 10, pp. 1445-1452, Oct. 2001

[26] H.-Y. Chen, L.-J. Deng, P.-H. Zhou, J. Xie, and Z.-W. Zhu, "Improvement of Surface Electromagnetic Waves Attenuation with Resistive Loading," Prog. Electromagn. Res. Lett., vol. 26, pp. 143152, 2011.

[27] F. Costa, S. Genovesi, A. Monorchio, and G. Manara, "Low-Cost Metamaterial Absorbers for Sub-GHz Wireless Systems," IEEE Antennas Wirel. Propag. Lett., vol. 13, pp. 27-30, 2014.

[28] C. Christopoulos, The transmission-line modeling method. Oxford University Press, 1995.

[29] G. T. Ruck, D. E. Barrick, W. D. Stuart, and C. K. Krichbaum, Radar cross section handbook, vol. 1. Plenum press New York, 1970.

[30] Y. Naito and K. Suetake, "Application of Ferrite to Electromagnetic Wave Absorber and its Characteristics," IEEE Trans. Microw. Theory Tech., vol. 19, no. 1, pp. 65-72, Jan. 1971.

[31] H. M. Musal and H. T. Hahn, "Thin-layer electromagnetic absorber design," IEEE Trans. Magn., vol. 25, no. 5, pp. 3851-3853, Sep. 1989.

[32] E. F. Knott and C. D. Lunden, "The two-sheet capacitive Jaumann absorber," IEEE Trans. Antennas Propag., vol. 43, no. 11, pp. 1339-1343, Nov. 1995.

[33] B. A. Munk, Frequency Selective Surfaces: Theory and Design. John Wiley \& Sons, 2005.

[34] F. Costa, A. Monorchio, and G. Manara, "Analysis and design of ultra thin electromagnetic absorbers comprising resistively loaded high impedance surfaces," IEEE Trans. Antennas Propag., vol. 58, no. 5, pp. 1551-1558, 2010.

[35] R. L. Fante and M. T. McCormack, "Reflection properties of the Salisbury screen," IEEE Trans. Antennas Propag., vol. 36, no. 10, pp. $1443-1454$, Oct. 1988

[36] F. Costa and A. Monorchio, "Closed-form analysis of reflection losses in microstrip reflectarray antennas," IEEE Trans. Antennas Propag., vol. 60, no. 10, pp. 4650-4660, 2012.

[37] F. Costa, S. Genovesi, A. Monorchio, and G. Manara, "A circuitbased model for the interpretation of perfect metamaterial absorbers," IEEE Trans. Antennas Propag., vol. 61, no. 3, pp. 12011209, 2013.
[38] B. Chambers, "Optimum design of a Salisbury screen radar absorber," Electron. Lett., vol. 30, no. 16, pp. 1353-1354, Aug. 1994.

[39] D. S. Weile, E. Michielssen, and D. E. Goldberg, "Genetic algorithm design of Pareto optimal broadband microwave absorbers," IEEE Trans. Electromagn. Compat., vol. 38, no. 3, pp. 518-525, Aug. 1996.

[40] E. Michielssen, J. M. Sajer, and R. Mittra, "Pareto-optimal design of broadband microwave absorbers using genetic algorithms," in Antennas and Propagation Society International Symposium, 1993. AP-S. Digest, 1993, pp. 1167-1170 vol.3.

[41] Eccosorb products, "http://www.eccosorb.com/productsoverview.htm." 02-Apr-2015.

[42] TDK Electromagnetic Absorbers, "http://www.emci.ru/f/rpm.pdf." 02-Apr-2015.

[43] D. M. Pozar, Microwave engineering. John Wiley \& Sons, 2009

[44] D. Micheli, R. Pastore, C. Apollo, M. Marchetti, G. Gradoni, V. M. Primiani, and F. Moglie, "Broadband Electromagnetic Absorbers Using Carbon Nanostructure-Based Composites," IEEE Trans. Microw. Theory Tech., vol. 59, no. 10, pp. 2633-2646, Oct. 2011.

[45] F. Moglie, D. Micheli, S. Laurenzi, M. Marchetti, and V. Mariani Primiani, "Electromagnetic shielding performance of carbon foams," Carbon, vol. 50, no. 5, pp. 1972-1980, Apr. 2012.

[46] Emerson a\& Cuming, "Basic principle of a graded absorber," http://www.eccosorb.eu/basic-principle-graded-absorber, 02-May2015.

[47] D. J. Kern and D. H. Werner, "A genetic algorithm approach to the design of ultra-thin electromagnetic bandgap absorbers," Microw. Opt. Technol. Lett., vol. 38, no. 1, pp. 61-64, Jul. 2003.

[48] N. I. Landy, S. Sajuyigbe, J. J. Mock, D. R. Smith, and W. J. Padilla, "Perfect metamaterial absorber," Phys. Rev. Lett., vol. 100, no. 20, p. 207402, 2008.

[49] Y. Zhang, R. Mittra, B.-Z. Wang, and N.-T. Huang, "AMCs for ultra-thin and broadband RAM design," Electron. Lett., vol. 45, no. 10, p. 484, 2009.

[50] T. Kollatou and C. Christopoulos, "Use of High-Impedance Surfaces in Electromagnetic Compatibility Applications," IEEE Trans. Magn., vol. 45, no. 3, pp. 1812-1815, Mar. 2009.

[51] F. Costa, S. Genovesi, and A. Monorchio, "On the bandwidth of high-impedance frequency selective surfaces," Antennas Wirel. Propag. Lett. IEEE, vol. 8, pp. 1341-1344, 2009.

[52] F. Costa and A. Monorchio, "Electromagnetic Absorbers based on High-Impedance Surfaces: From ultra-narrowband to ultrawideband absorption," Adv. Electromagn., vol. 1, no. 3, pp. 7-12, 2012.

[53] B. A. Munk, P. Munk, and J. Pryor, "On designing Jaumann and circuit analog absorbers (CA absorbers) for oblique angle of incidence," IEEE Trans. Antennas Propag., vol. 55, no. 1, pp. 186193, 2007.

[54] S. W. Simms and V. F. Fusco, "Thin Radar Absorber Using an Artificial Magnetic Ground Plane," in Microwave Conference, 2006. 36th European, 2006, pp. 1167-1169.

[55] H. Mosallaei and K. Sarabandi, "A one-layer ultra-thin meta-surface absorber," in 2005 IEEE Antennas and Propagation Society International Symposium, 2005, vol. 1B, pp. 615-618 vol. 1B.

[56] H.-B. Zhang, P.-H. Zhou, H.-P. Lu, Y.-Q. Xu, D.-F. Liang, and L.-J. Deng, "Resistance selection of high impedance surface absorbers for perfect and broadband absorption," IEEE Trans. Antennas Propag., vol. 61, no. 2, pp. 976-979, 2013.

[57] O. Luukkonen, C. Simovski, G. Granet, G. Goussetis, D. Lioubtchenko, A. V. Raisanen, and S. A. Tretyakov, "Simple and accurate analytical model of planar grids and high-impedance surfaces comprising metal strips or patches," IEEE Trans. Antennas Propag., vol. 56, no. 6, pp. 1624-1632, 2008.

[58] K. N. Rozanov, "Ultimate thickness to bandwidth ratio of radar absorbers," IEEE Trans. Antennas Propag., vol. 48, no. 8, pp. 1230$1234,2000$.

[59] D. Ragle and S. Kayali, "Hydrogen effects on GaAs microwave semiconductors," in GaAs Reliability Workshop, 1997., Proceedings, 1997, pp. 66-71.

[60] N. Marcuvitz, Waveguide handbook. Iet, 1951.

[61] M. G. Vigueras, J. L. G. Tornero, G. Goussetis, and A. A. Melcon, "Software tool for the leaky-mode analysis of waveguides loaded 
IEEE Electromagnetic Compatibility (EMC) Magazine, PREPRINT

with frequency selective surfaces," in 3rd European Conference on

Antennas and Propagation, 2009. EuCAP 2009, 2009, pp. 83-87. 